\title{
Sensitivity Analysis of Active Flow Control Systems: Operating Parameters and Configuration Design
}

\author{
Mark Jabbal ${ }^{\star}$ and Valerio Tomasso ${ }^{\dagger}$ \\ Brunel University, London UB8 3PH, United Kingdom
}

\section{Nomenclature}

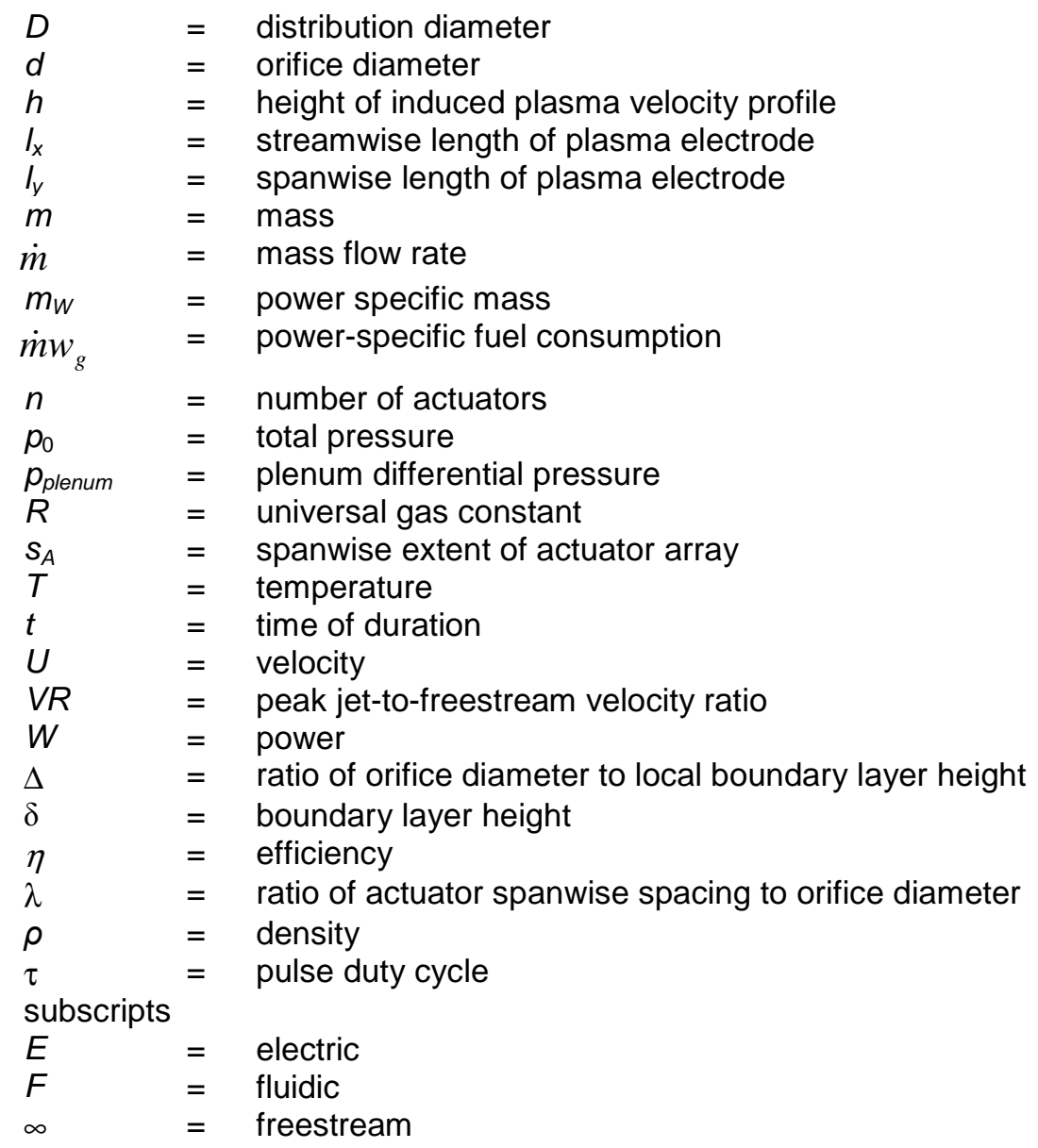

\section{Introduction}

Aerodynamic gains offered by active flow control (AFC) can be maximized if AFC system design is included as part of the initial aircraft optimization process. In the more likely scenario where inception of AFC systems is initially via retrofitting to existing aircraft platforms, implementation will largely be dictated by whether such

\footnotetext{
*Lecturer, School of Engineering and Design, Kingston Lane, Uxbridge. Corresponding author, Email: mark_jabbal@hotmail.com

'Student, School of Engineering and Design, Kingston Lane, Uxbridge.
} 
systems can be installed within the available mass, power and space constraints. Research has been undertaken to understand the nature of systems architectures needed to support the generation, management, and distribution of power to AFC actuators for application on commercial transport aircraft ${ }^{1}$. The motivation for this study is to understand how current AFC systems meet the constraints imposed by an A320 aircraft and the extent to which variations in system configuration, operating parameters and efficiency affect overall system mass and power consumption.

There is still considerable uncertainty with regards to AFC system design for compliance with aircraft constraints. Consider the example of fluidic actuators, which can take the form of pulsed jets ${ }^{2}$, vortex generating jets ${ }^{3}$ or sweeping jets ${ }^{4}$, and which can be used for separation control applications in off-design flight conditions. Power to the actuators can be supplied pneumatically via direct engine bleed or electrically and pneumatically via electrical air compressors (referred to hereon as the 'hybrid' system architecture ${ }^{1}$ ). The choice between distributing power electrically or pneumatically is however still an open question. Furthermore, there is the choice of whether a centralized compressor or a series of localized compressors should be utilized for the hybrid system, in addition to actuator operating parameters that satisfy performance and system requirements. For cruise flight, plasma actuators have been proposed for skin friction drag reduction applications $^{5}$ as part an 'electro-fluidic' system architecture ${ }^{1}$. Current actuator efficiency of dielectric barrier discharge (DBD) plasmas is however very low, typically $0.1 \%{ }^{5}$ (based on the conversion of electrical input power to 'mechanical' output power, namely induced kinetic energy density flow rate measured from local velocity profiles), with the consequence of excessively high system mass and power costs ${ }^{1}$. Thus, it is useful to know the gains in efficiency that will make implementation viable.

Within the present work it is proposed that the sensitivity analyses conducted against the backdrop of applicable design trades and existing A320 system hardware data ${ }^{1}$, will show the extent to which aircraft constraints can be met, as well as inform the wider flow control community on viable strategies for AFC systems implementation and targeted improvements in actuator efficiency.

\section{Research Methodology}

\section{A. AFC System Modeling}


The present work uses a scalable, low-order system mass model ${ }^{1}$, as defined in Eq. (1).

$$
m=\frac{W_{F}}{\eta}\left[\Sigma m_{w}(\eta)+\dot{m}_{W g} t\right]
$$

The overall system mass, $m$, is equal to sum of the AFC system hardware masses and the mass cost of the energy used by the system. The former is made up of scalable power specific mass terms, $m_{w}$, for each of the generation, management, distribution and actuation subsystems that constitute to the systems architecture; whereas the latter is the mass of the fuel used for the duration of AFC operation and is determined from the power-specific fuel consumption of the generator system, $\dot{m} w_{g}$. Both $m_{w}$ and $\dot{m} w_{g}$ have units of $\mathrm{kg} / \mathrm{kW}$ and are multiplied in Eq. (1) by the overall system power consumption, $W_{E}$ (equivalent to the fluidic output power from an array of AFC actuators, $W_{F}$, divided by overall power efficiency of the system, $\eta$ ) to give the overall system mass. Commercial, aerospace-specific data for pneumatic ducts $^{\ddagger}$ and electric wires ${ }^{\S}$ are used to compare the relative benefits of distributing power pneumatically and electrically, and subsequently added to Eq. (1) in the form of a distribution $m_{w}$ term for a given AFC application.

The constraint for AFC system mass in this study is provided by Airbus UK design trades, which indicates that for a $1 \%$ overall drag reduction delivered by an AFC system to an A320 aircraft, maximum AFC system mass permitted is $250 \mathrm{~kg}\left(\sim 0.4 \%\right.$ of Operating Empty Weight, OEW) ${ }^{* *}$. Thus, it is assumed that each AFC system considered is capable of delivering the stated drag reduction within the mass limit. Available power for the AFC system is limited by the number of engine integrated drive generators (IDG); for the A320 there are two IDGs which deliver $90 \mathrm{~kW}$ each. It is also assumed that the largest duct/cable diameter permitted is that which can be accommodated in the A320 wing leading edge; i.e. approximately $50 \mathrm{~mm}^{1}$. All other A320 related systems, including AFC power generation and management subsystems have been previously documented ${ }^{1}$.

\section{B. Data Reduction}

\section{Hybrid System Architecture}

Utilization of the hybrid system architecture with pulsed-air jet actuators ${ }^{2}$ can make use of a centralized or decentralized compressor configuration, as illustrated in Figure 1. The centralized configuration uses a macro-

\footnotetext{
¥Data available online at: http://flexfab.com/wp-content/uploads/2012/12/Dist-Catalog-12-21-12.pdf [retrieved 6 Jun. 2013].

$\S$ Data available online at: http://www.wirefacts.com/MIL-W-2275934.php [retrieved 4 Sep. 2012].

** Private communication, Airbus Operations Ltd, 2010.
} 
scale compressor, whereas the decentralized configuration uses meso- or micro-scale compressors. Each configuration was compared for LE slat separation control application, of which the actuator implementation parameters are shown in Table 1. Based on compressor mass, power rating and efficiency, $m_{W}$ terms are obtained for each configuration for input in Eq. (1) to deduce system costs. Furthermore, individual compressor mass flow rate is compared against total mass flow rate requirement (Table 1) to deduce the number of actuators per compressor:

1) Micro-scale $(8 \mathrm{~mm} ; 0.36 \mathrm{~g} / \mathrm{s} ; ~ 5 \% \text { efficiency })^{6}-m_{W}=18.2 \mathrm{~kg} / \mathrm{kW} ; 17$ actuators per compressor

2) Meso-scale $(25 \mathrm{~mm} ; 2.4 \mathrm{~g} / \mathrm{s} ; ~ 50 \% \text { efficiency })^{7}-m_{W}=1.82 \mathrm{~kg} / \mathrm{kW} ; 115$ actuators per compressor

3) Macro-scale (200mm; 53g/s; $85 \%$ efficiency) ${ }^{1}-m_{W}=1.07 \mathrm{~kg} / \mathrm{kW} ; 2530$ actuators per compressor

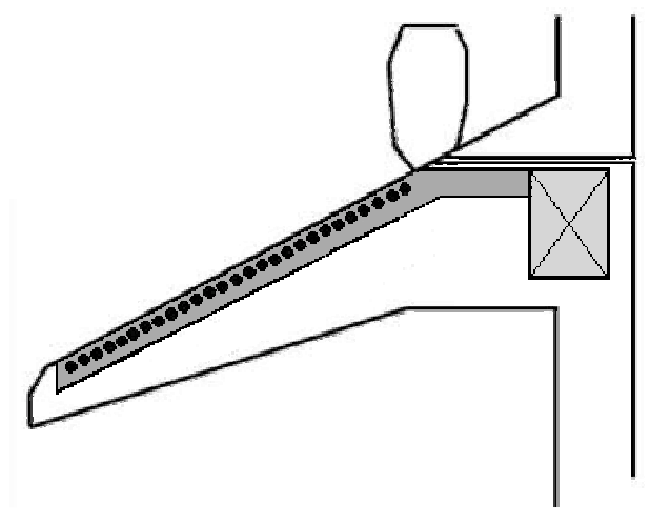

a) Macro-scale compressor system

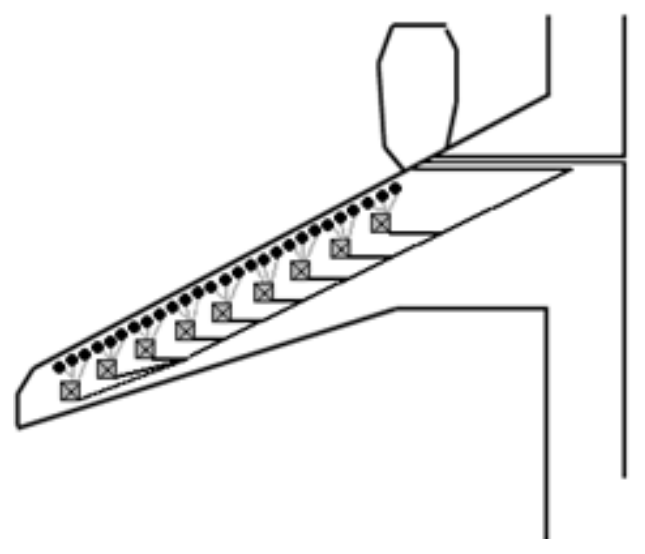

b) Meso-scale compressor system

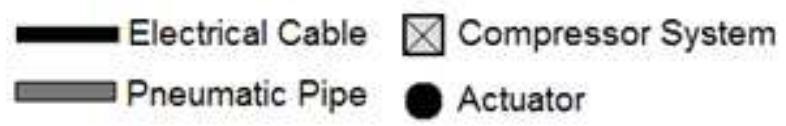

Fig. 1 Macro-scale and meso-scale compressor configurations for the hybrid system architecture 
Table 1 Air-jet actuator implementation parameters for LE slat separation control

\begin{tabular}{llllll}
\hline \hline $\begin{array}{l}\text { Reference } \\
\text { parameters }\end{array}$ & \multicolumn{2}{l}{$\begin{array}{l}\text { Actuator design } \\
\text { parameters }\end{array}$} & \multicolumn{2}{l}{$\begin{array}{l}\text { Output } \\
\text { parameters }\end{array}$} \\
\hline$U_{\infty}$ & $340 \mathrm{~m} / \mathrm{s}$ & $\Delta$ & 0.2 & $n$ & 6426 \\
$\delta$ & $2 \mathrm{~mm}$ & $\lambda$ & 10 & $d$ & $0.5 \mathrm{~mm}$ \\
$\rho$ & $1 \mathrm{~kg} / \mathrm{m}^{3}$ & $\mathrm{~s}_{\mathrm{A}}$ & $30 \mathrm{~m}$ & $U_{\text {jet }}$ & $340 \mathrm{~m} / \mathrm{s}$ \\
$V R$ & 1 & $m_{W}$ & $2 \mathrm{~kg} / \mathrm{kW}$ & $p_{\text {plenum }}$ & $234 \mathrm{kPa}$ \\
$\tau$ & $25 \%$ & $\eta_{\text {orifice }}$ & $\sim 40 \%$ & $\dot{m}$ & $0.11 \mathrm{~kg} / \mathrm{s}$ \\
$t$ & $600 \mathrm{~s}$ & - & - & - & - \\
\hline \hline
\end{tabular}

To examine actuator parameter sensitivity on hybrid system mass, power consumption and distribution diameter requirements, velocity ratio, $V R$, and pulse duty cycle, $\tau$ were varied. $V R$ represents the ratio of actuator peak exit velocity to local freestream velocity and $\tau$ is defined as the ratio between the time the actuator is on and off. These two parameters dictate total fluid power delivered by an actuator array ${ }^{1}$, as shown in Eq. (2)

$$
W_{F}=\tau \frac{\pi}{4} \frac{(V R)^{3} \Delta}{\lambda} \frac{1}{2} \rho s_{A} \delta U_{\infty}^{3}
$$

Duct diameter sizing for a given AFC application is determined by the mass flow rate and air-jet plenum pressure $^{8}$ (where flow velocity in the duct is limited to Mach 0.2 , based on safe industrial practice to mitigate against duct fatigue and possible rupture)

$$
D=4 \pi \frac{\dot{m} R T}{U\left(p_{\text {plenum }}+p_{0}\right)}
$$

\section{Electro-Fluidic System Architecture}

To examine actuator efficiency sensitivity on system mass and power consumption, the efficiency term, $\eta$ was varied in Eq. (1) under the assumption that the inherently low actuator efficiency $(\sim 0.1 \%)$ and small variation in the present study $(<1 \%)$ was sufficient to render other subsystem efficiencies, which are much larger, as negligible. The study was conducted for skin friction drag reduction application along the wing main element, with implementation parameters shown in Table 2. 
Table 2 DBD plasma actuator implementation parameters for main element skin friction drag reduction

\begin{tabular}{llllll}
\hline \hline $\begin{array}{l}\text { Reference } \\
\text { parameters }\end{array}$ & \multicolumn{2}{l}{$\begin{array}{l}\text { Actuator design } \\
\text { parameters }\end{array}$} & \multicolumn{2}{l}{$\begin{array}{l}\text { Output } \\
\text { parameters }\end{array}$} \\
\hline$U_{\infty}$ & $265 \mathrm{~m} / \mathrm{s}$ & $I_{x}$ & $1 \mathrm{~m}$ & $n$ & 750 \\
$\delta$ & $14 \mathrm{~mm}$ & $I_{y}$ & $2 \mathrm{~mm}$ & $U_{\text {jet }}$ & $\sim 10 \mathrm{~m} / \mathrm{s}$ \\
$\rho$ & $0.5 \mathrm{~kg} / \mathrm{m}^{3}$ & $\mathrm{~S}_{\mathrm{A}}$ & $30 \mathrm{~m}$ & $h$ & $2 \mathrm{~mm}$ \\
$V R$ & 0.05 & $m_{W}$ & $0.03 \mathrm{~kg} / \mathrm{kW}$ & $V_{\mathrm{ac}}$ & $25 \mathrm{kV}$ \\
$t$ & $4080 \mathrm{~s}$ & - & - & - & - \\
\hline \hline
\end{tabular}

A $1 \mathrm{~m}$ chordwise electrode length corresponds to $30 \%$ wing area coverage. The output power of plasma actuators $^{1}$ for local fluid acceleration is given by Eq. (4) and is used in Eq. (1) to determine system costs

$$
W_{F}=\frac{1}{6} \frac{(V R)^{3}}{\lambda} \rho s_{A} h \frac{l_{x}}{l_{y}} U_{\infty}^{3}
$$

\section{Results}

\section{A. Distribution Systems: Comparison of Pneumatic and Electric Power Distribution Methods}

Figure 2 compares power specific mass, distribution diameter and efficiency as a function of power transmission for electric and pneumatic distribution in a wire and duct respectively. Below 20kW, distribution power specific mass, diameter and efficiency are all markedly lower for electric distribution. Above 20kW, power specific mass is lower for pneumatic distribution, although in practice it would be more mass efficient to transmit high electrical power through a bundle of smaller cables than a single, large one due to insulation and heat transfer issues. Mass and efficiency progressively increase for pneumatic distribution at high power, however duct diameter is limited at $50 \mathrm{~mm}$ (corresponding to $60 \mathrm{~kW}$ transmission) by internal wing constraints. Electric distribution is therefore the only viable option above $60 \mathrm{~kW}$. Thus from a practical perspective, there is clear benefit to utilizing electrical power distribution as the basis for AFC implementation on aircraft. 


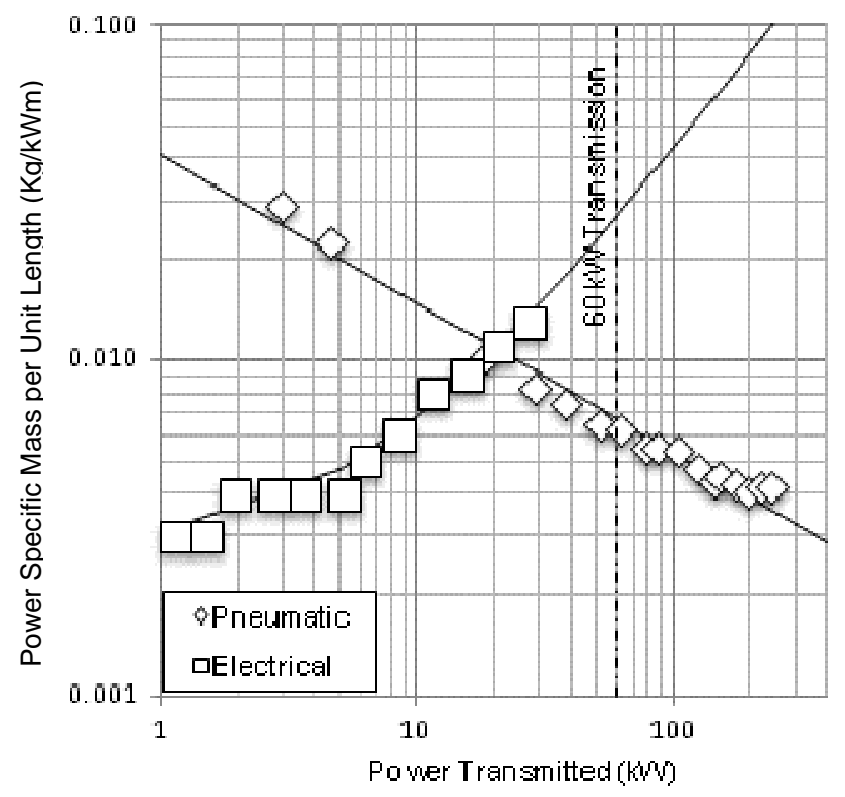

a) Distribution power specific mass per unit length

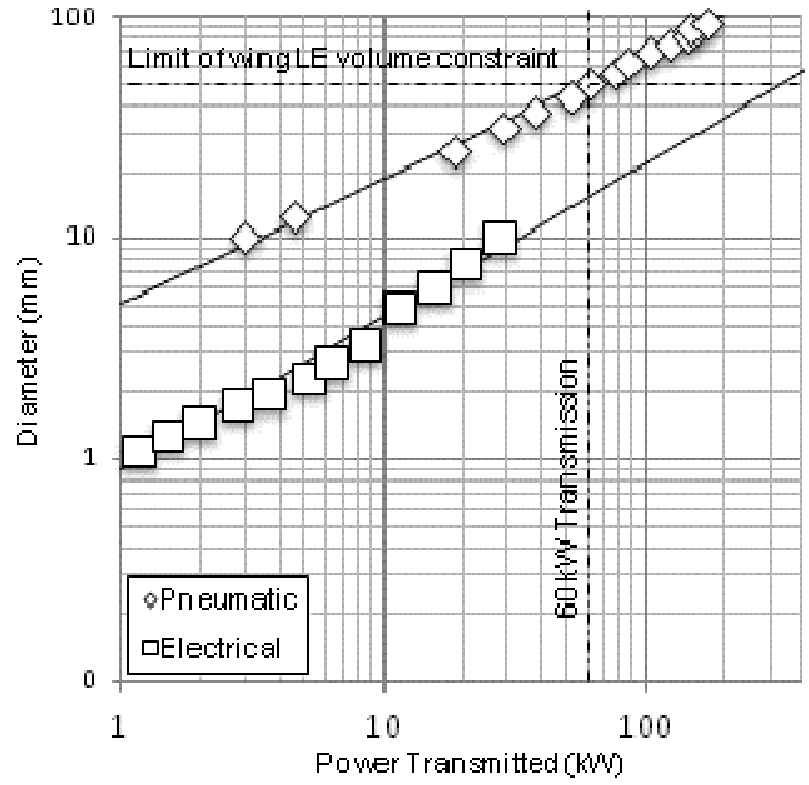

b) Power distribution diameter 


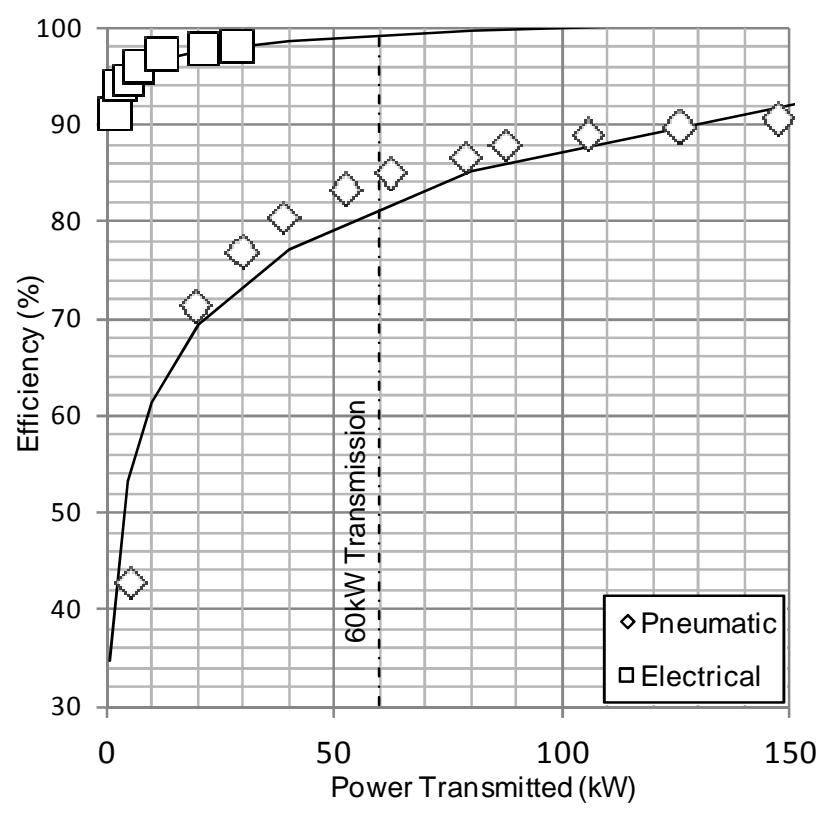

c) Power distribution efficiency

Fig. 2 Variation of power distribution characteristics with power transmission

\section{B. Generation Systems: Comparison of Micro-, Meso- and Macro-scale Compressor Configurations on the Hybrid System Architecture}

Comparison of power specific mass for the hybrid AFC system architecture with micro-, meso- and macroscale compressor arrangements is shown in Fig. 3a. The power specific mass for the micro compressor configuration is approximately $20 \mathrm{~kg} / \mathrm{kW}$; i.e. $20 \mathrm{~kg}$ of system hardware required for $1 \mathrm{~kW}$ of power flow through that system. This is relatively high compared to both the meso and macro configurations $(\sim 4 \mathrm{~kg} / \mathrm{kW})$ and is due to the inherently low compressor efficiency $(\sim 5 \%)$. Consequently, over $85 \%$ of the system mass in the micro compressor configuration is required in power generation.

Figure 3b compares absolute system mass of each configuration for LE slat separation control (Table 1). The high system cost of the micro compressor configuration is confirmed with an overall mass which exceeds constraints by $75 \mathrm{~kg}$. Both meso- and macro-scale configurations each have a system mass under the constraint, with the former approximately $13 \mathrm{~kg}$ greater than the latter. From a practical perspective however, this small mass penalty may be offset by the potentially wider benefits of the meso-scale configuration. The macro-scale configuration features a relatively large $(200 \mathrm{~mm}$ diameter) centralized compressor with a $22 \mathrm{~mm}$ diameter main duct supplying air to the actuators (Fig. 1a). This is in contrast to the meso-scale configuration 
featuring multiple compressors (25mm diameter) with $3 \mathrm{~mm}$ diameter ducts feeding the actuators (Fig. 1b) and which therefore has a smaller form factor conducive for aircraft implementation. The decentralized nature of the meso-scale configuration also ensures that potential compressor failure will remain localized, thus minimizing significant loss in overall AFC performance.

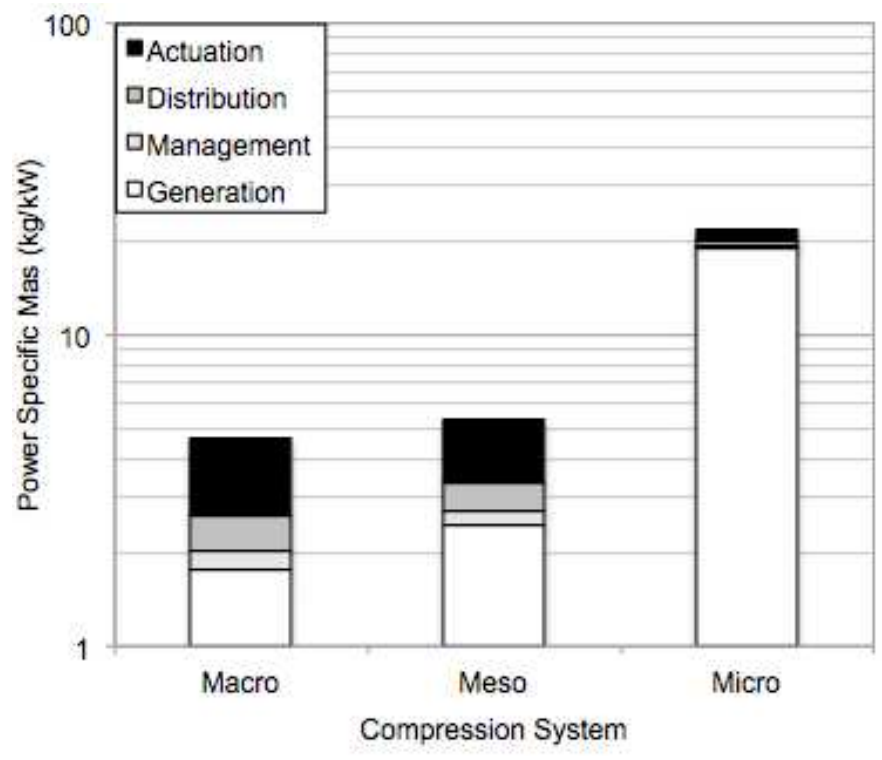

a) Power specific mass

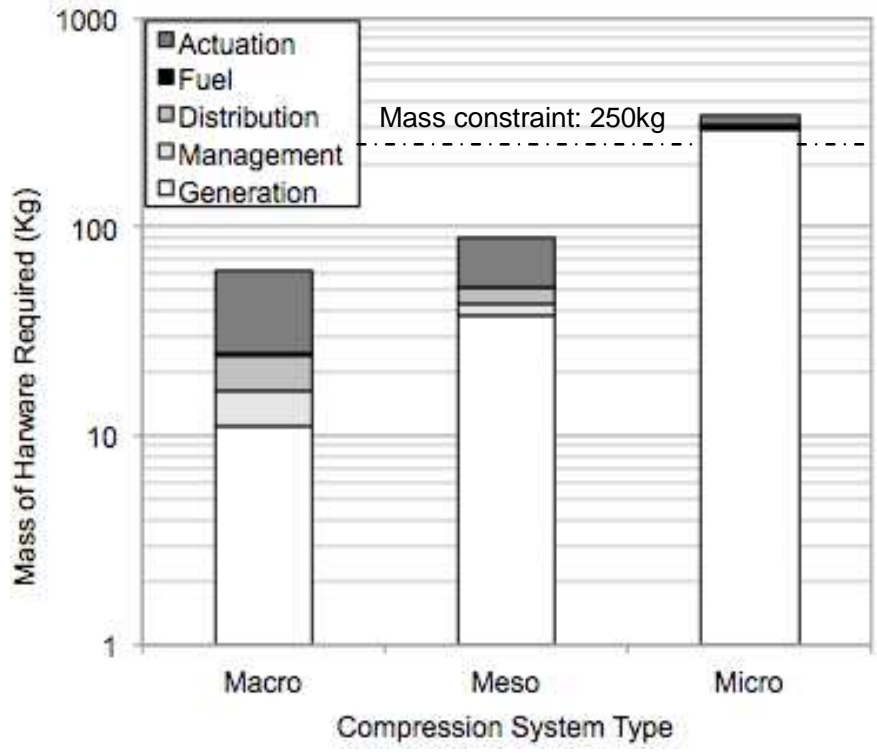

b) System mass

Fig. 3 Comparison of relative subsystem and absolute architecture system mass for macro-, mesoand micro-scale compressor configurations. 


\section{Actuator Systems: Parameter Sensitivity of Air-jet Actuators on the Hybrid System Architecture}

The effect of jet-to-freestream velocity ratio, $V R$ and pulse duty cycle, $\tau$ on the hybrid AFC system architecture mass, power consumption and distribution diameter are shown in Fig. 4. Results presented are based on the macro compressor configuration for the application defined in §B.1. In Fig. 4a, the magnitude of system mass increases with both $V R$ and $\tau$, with the rate of increase greater for $V R$, in accordance with Eq. (2). At $\tau=100 \%$, i.e. steady blowing, system mass drops as the need for an actuator pulse valve system and its associated electrical supply becomes redundant and hence is omitted. Use of fluidically pulsed jets such as sweeping jet actuators ${ }^{4}$ would eliminate valves completely and thus reduce mass requirements. The system remains below the mass limit when operated at $V R=0.5$ and 1.0 for all values of $\tau$, but exceeds the limit when operating at $V R=1.5$ for $\tau>30 \%$. Similar trends are observed with system power consumption (Fig. 4b), with power constraint from a single IDG exceeded at $V R=1.5$ for $\tau>50 \%$ and maximum power constraint (two IDGs) exceeded for $V R=1.5$ for $\tau>90 \%$.

Contrary to the trends shown in system mass and power consumption, it is observed in Fig. 4c that duct diameter is inversely proportional to $V R$ and directly proportional to $\tau$. This is due to the relation defined in Eq. (3). Diameter constraint is only exceeded for steady blowing conditions at $V R=0.5$.

In a practical context, a hybrid system designed to operate air-jet actuators at $V R=1$ offers the most versatility by permitting operation across the full range of $\tau$ without exceeding mass and power constraints associated with higher VR operation, or duct diameter constraint for lower VR operation. It also suggests that 'brute force' techniques $^{9}$ for separation control with $V R>>1$ (i.e. tangential blowing) are not suitable for aircraft implementation via the hybrid architecture, unless utilized for smaller areas of application or are capable of delivering substantially larger gains in drag reduction to offset the higher system costs. 


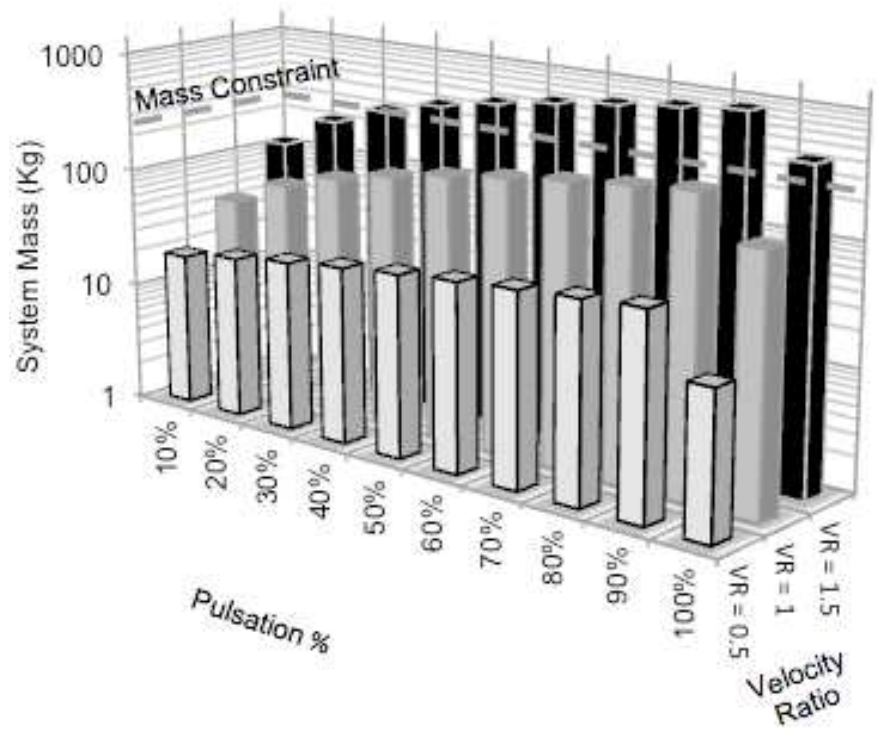

a) System mass

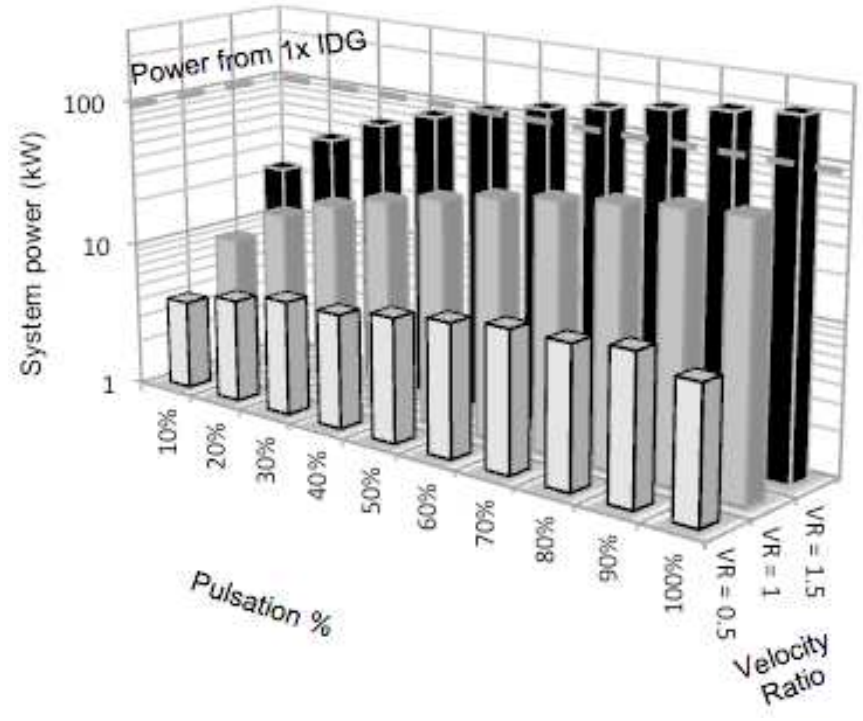

b) System power consumption 


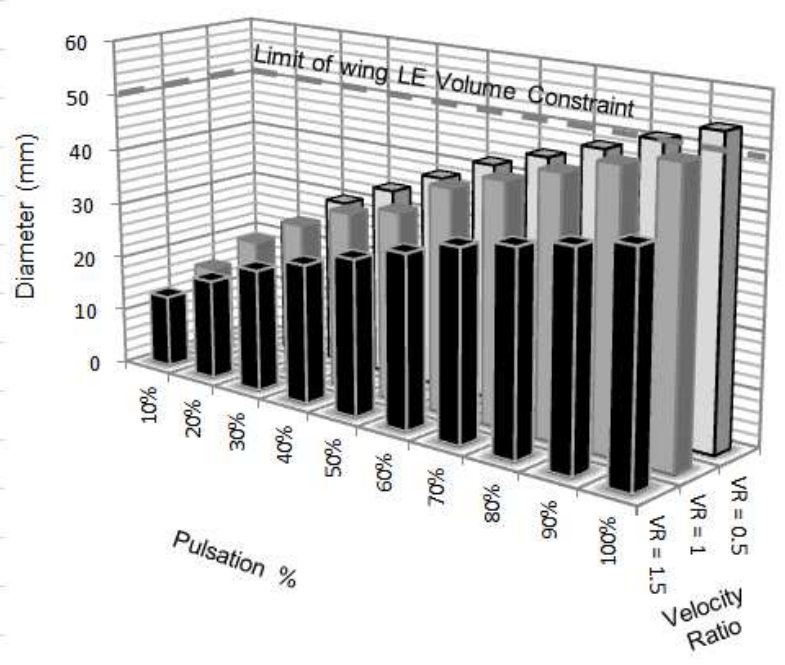

c) System duct diameter

Fig. 4 Variation of hybrid architecture systems costs with air-jet actuator operational parameters

\section{Actuator Systems: Efficiency Sensitivity of DBD Plasma Actuators on the Electro-fluidic System Architecture}

The effect of plasma actuator efficiency on system power consumption and mass of the electro-fluidic AFC architecture is presented in Fig. 5 for wing main element skin friction drag reduction (Table 2). Based on current actuator efficiency ${ }^{5} \sim 0.1 \%$, power consumption and mass exceed constraints by $110 \mathrm{~kW}$ and $180 \mathrm{~kg}$ respectively. To satisfy maximum power constraints for the same chordwise electrode length, actuator efficiency should be increased to $0.16 \%$, which equates to a system mass also just within the maximum limit. A threefold increase in efficiency to $0.32 \%$ would lower power consumption to that producible by a single IDG, while lowering system mass $120 \mathrm{~kg}$ below the constraint. 


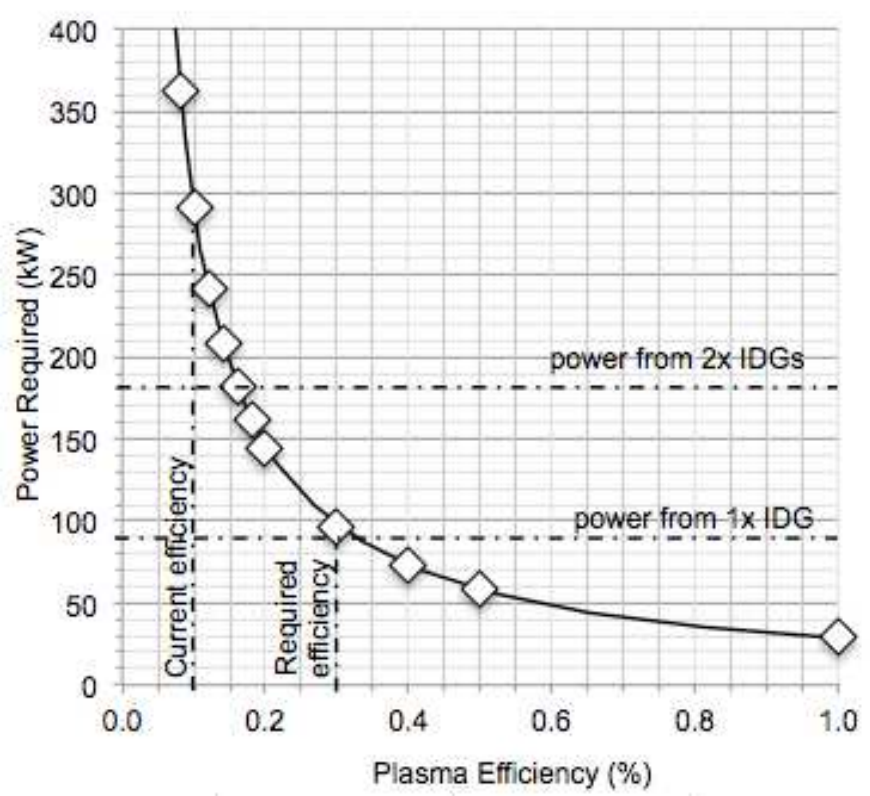

a) System power consumption

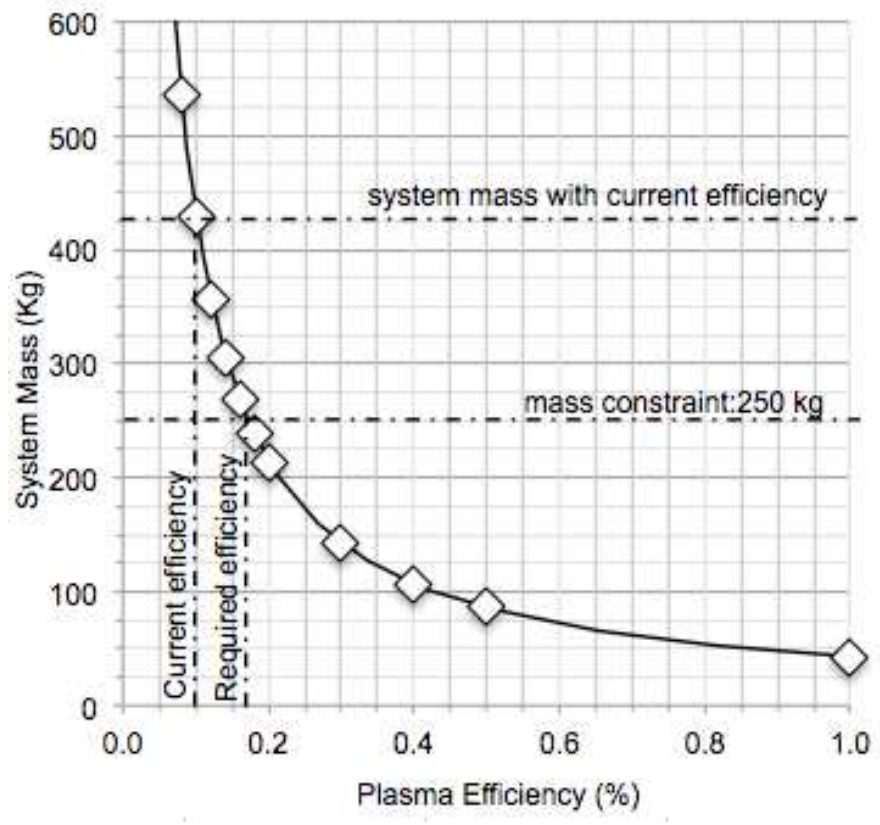

b) System mass

Fig. 5 Variation of electro-fluidic architecture systems costs with plasma actuator efficiency for chordwise electrode length of $1 \mathrm{~m}$ 


\section{Conclusions}

This paper has outlined sensitivity analyses for AFC systems architectures to understand how the method of power distribution, compressor scale for power generation, and actuator operational parameters and efficiency affect overall system mass and power consumption. The work was conducted with an existing AFC system mass model ${ }^{1}$ applied to an A320 aircraft under consideration of relevant AFC design trades and engineering constraints.

The major findings of this study are summarized as follows:

1) For power transmission $<20 \mathrm{~kW}$, electrical power distribution is more efficient than pneumatic power distribution (less by a factor of 2 at $5 \mathrm{~kW}$ ) and more cost effective in mass and diameter/space constraints (less by a factor of 4 and 5 respectively at $5 \mathrm{~kW}$ ). For power transmission $>20 \mathrm{~kW}$, pneumatic distribution is more competitive in mass (less by a factor of 3.5 at $50 \mathrm{~kW}$ ), but becomes impractical above $60 \mathrm{~kW}$ as duct diameter exceeds the physical constraint of the wing leading edge volume. These observations suggest that AFC systems architectures should be based on electrical power distribution, with the implication of utilizing electrically-powered AFC actuators or in the case of fluidic actuators, electrically-powered air compressors ('hybrid' system) rather than direct engine bleed.

2) The hybrid AFC architecture, incorporating air-jet actuators with electrically-powered compressors, shows that for LE slat separation control the micro-scale compressor configuration exceeds maximum permitted system mass by $75 \mathrm{~kg}$. Both meso-scale and macro-scale compressor configurations satisfy mass constraints, with the former approximately $13 \mathrm{~kg}$ greater than the latter. However the greater practicalities conferred by the meso-scale compressor configuration, such as a smaller form factor, fault isolation and smaller consequence of compressor failure, make it the most viable strategy for hybrid AFC implementation.

3) Operational parameter sensitivity of air-jet actuators on the hybrid AFC architecture shows that system mass and power consumption are proportional to both jet-to-freestream velocity ratio $(V R)$ and pulse duty cycle $(\tau)$, whereas distribution diameter is inversely proportional to $V R$. For the range of $V R(0.5,1.0,1.5)$ and $\tau(10 \%-100 \%)$ investigated for LE slat separation control, system mass and power constraints (based on single IDG output) requires $\tau<30 \%$ at $V R=1.5$, with $V R=1$ ensuring operation across full range of $\tau$. 
4) For the electro-fluidic AFC architecture incorporating a DBD plasma actuator array along the wing main element with $1 \mathrm{~m}$ chordwise electrode length, system mass and power constraints are exceeded by $180 \mathrm{~kg}$ and $110 \mathrm{~kW}$ respectively based on current actuator efficiency $(0.1 \%)$. To make the AFC system compliant with mass and maximum power constraints (based on total IDG output) for the same chordwise length, actuator efficiency needs to be increased to $0.16 \%$. A threefold improvement in plasma efficiency $(0.32 \%)$ would make the AFC system compliant with single IDG output power constraint, while affording a reduced mass (120kg below mass constraint).

The above results should be placed in the context that actuators and micro/meso-scale compressors are still under research and development and that the prospect of further gains in performance can be expected, which will lead to lower overall systems weight and thus greater viability for aircraft integration. From the results of this study the authors anticipate that the application of fluidic actuators as part of a decentralized compressor system for separation control to be a realizable goal in the near term, whereas the application of plasma actuators for viscous drag reduction remains a much longer term goal.

Finally, a note on the AFC systems cost/performance design trade. Whilst drag reduction is a suitable metric for plasma actuators aimed at long duration (cruise) applications, an alternative metric for fluidic actuators aimed at short duration (take-off and landing) applications may be more suitable, e.g. permitted systems mass for percentage of lift enhancement or increase in $\mathrm{C}_{\text {Lmax }}$. It should also be noted that while larger aircraft will permit larger AFC systems costs to be incurred, the relationship between AFC system mass and aircraft size (e.g. OEW) is not a linear one. As such, similar studies for other aircraft should be carried out on a case-bycase basis using separate design trades.

\section{References}

[1] Jabbal, M., Liddle, S.C., and Crowther, W.J., "Active Flow Control Systems Architectures for Civil Transport Aircraft," Journal of Aircraft, Vo. 47, No. 6, 2010, pp. 1966-1981.

doi: $10.2514 / 1.52842$ 
[2] Warsop, C., Hucker, M., Press, A.J., and Dawson, P., "Pulsed-Air Jet Actuators for Flow Separation Control," Flow, Turbulence and Combustion, Vol. 78, No. 3-4, 2007, pp. 255-281.

doi: 10.1007/s10494-006-9060-4

[3] Godard, G., Foucaut, J.M. and Stanislas, M., "Control of a Decelerating Boundary Layer. Part 2: Optimization of Slotted Jet Vortex Generators," Aerospace Science and Technology, Vol. 10, No. 5, 2006, pp. 394-400.

doi: 10.1016/j.ast.2005.11.006

[4] Woszidlo, R., Raghu, S., and Wygnanski, I., "Parametric Study of Sweeping Jet Actuators for Flow Separation Control," AIAA Paper 2010-4247, June 2010.

doi: $10.2514 / 6.2010-4247$

[5] Moreau, E., "Airflow Control by Non-Thermal Plasma Actuators," Journal of Physics D: Applied Physics, Vol. 40, Feb. 2007, pp. 605-636.

doi: $10.1088 / 0022-3727 / 40 / 3 / S 01$

[6] Epstein, A., "Millimeter-Scale, Micro-Electro-Mechanical Systems Gas Turbine Engines," Journal of Engineering for Gas Turbines and Power, Vol. 126, Apr. 2004, pp. 205-226.

doi:10.1115/1.1739245

[7] Kang, S., "Fabrication of Functional Mesoscopic Ceramic Parts for Micro Gas Turbine Engines," PhD Thesis, Stanford University, USA, 2001.

[8] Bray, T.P., and Garry, K.P., "Optimization of Air-Jet Vortex Generators with Respect to System Design Parameters," The Aeronautical Journal, Vol. 103, No. 1028, 1999, pp. 475-479.

[9] Warsop, C., "MEMS and Microsystems Technologies - Their Potential and Status for Drag Reduction and Separation Control," European Congress on Computational Methods in Applied Science and Engineering (ECCOMAS), July 2004. 